satisfactorily; it is necessary to find the correct exposure by trial and error.

While the copper grids must be handled carefully and given the minimum exposure compatible with complete clearing, the woven nickel grids are very robust and can be given quite long exposures without affecting their strength. In tests in this laboratory, such a grid selected at random from a box was held at white heat for two seconds and cooled ten times without showing signs of deterioration other than tarnishing of the surface.

The accompanying photograph shows the corner of a copper grid cleaned by this method.

Department of Experimental Pathology

G. Eaves

and Cancer Research

Medical School,

University of Leeds,

Leeds 2.

Jan. 31 .

\section{Influence of Structure on the Plastic Flow of Steel above the $A_{3}$-Point}

IN connexion with the work done on the plastic flow of steel above the $A_{2}$-point, a detailed account of which will be published elsewhere, it was desirable to determine whether the relationship between the temperature and flow-rate at a given stress, hitherto determined by means of a series of experiments, each involving the flow of a separate specimen at a different temperature, could not be deduced without appreciable error from a single experiment in which flow took place under conditions of rising temperature. This possibility was investigated in two similar experiments. In each the specimen was first annealed in vacuo at $950^{\circ}$ for approximately twenty minutes in situ. Then it was allowed to flow at that temperature under a constant tensile stress until the steady flow had definitely established itself, when the temperature was raised at an approximately constant rate from $950^{\circ}$ to $1,250^{\circ} \mathrm{C}$. in twelve minutes. The extension, time and temperature were recorded simultaneously. The natural strain-rates at temperatures in this range, which were deduced from these readings, were found to be considerably higher (upper curve) over most of the range than the corresponding values obtained under conditions of constant temperature.

In the flow at constant temperature ${ }^{1}$, specimens were annealed as before, prior to the application of the stress, each, however, at the same temperature at which it was ultimately allowed to flow. Within the stress and temperature regions $190-450 \mathrm{kgm}$. $\mathrm{cm} .{ }^{2}, 950-1,300^{\circ} \mathrm{C}$. respectively, the natural tensile strain-rate $\dot{\varepsilon}$ of the steady, quasi-viscous flow of plain 0.2 per cent carbon steel flowing under constant stress in vacuo was thus found to be given, within the limits of experimental error, by

$$
\dot{\varepsilon}=A \cdot T \cdot \exp (-H / k T) \cdot \sinh (q \sigma / k T) \text {, }
$$

where $A=6.6 \times 10^{-3}$ sec. ${ }^{-1} /{ }^{\circ} \mathrm{C}$., $\quad H / k=36.8 \times$ $10^{3}{ }^{\circ} \mathrm{C} ., q / k=22 \cdot 0 \mathrm{~cm} .{ }^{2} /{ }^{\circ} \mathrm{C}$. kgm., $\sigma$ being expressed in $\mathrm{kgm} . / \mathrm{cm} .^{2}$ and $\dot{\varepsilon}$ in sec. ${ }^{-1}$. Experimental points, obtained with specimens flowing under a stress of $197 \mathrm{kgm} . / \mathrm{cm} .{ }^{2}$, denoted by black dots, together with the (lower) curve derived from the above relation, are shown in the graph.

The conclusion is reached that, within the given ranges of the variables, the influence of the initial

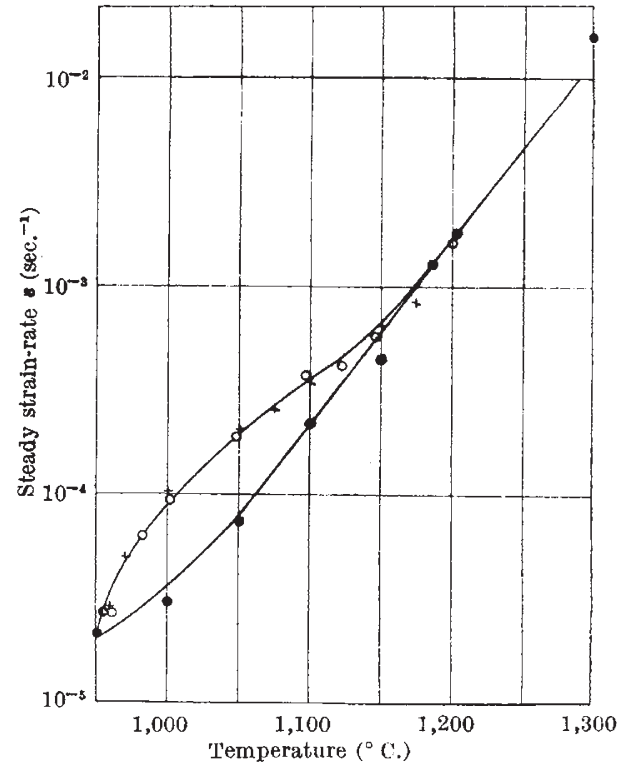

Plastic flow of 0.2 per cent carbon steel at constant stress of $197 \mathrm{kgm} . / \mathrm{cm} .^{2}:$ (1)
(2) $\times-\times-$, flow at rising temperature ; (3) $0-0-$, repeat of (2)

state of the metal on the flow precludes the possibility of a simple derivation of steady strain-rates of specimens annealed at the flow-temperature by means of data obtained from the flow under conditions of varying temperature. The origin of the discrepancies presumably lies in the non-attainment at all temperatures in the range, in specimens flowing under conditions of varying temperature, of a characteristic grain-size, such as is attained in individual specimens annealed at the temperature of $\mathrm{flow}^{2,3}$. However, the observed ultimate coalescence of both curves indicates that the second method might fulfil its intended purpose outside the range of variables so far investigated.

Physics Laboratories,

P. Feltham

British Iron and Steel Research Association, Battersea,

London, S.W.I1.

Jan. 1.

${ }^{1}$ Feltham, P., Metal Treatment (Oct. 1951).

${ }^{2}$ Cleaves, H. E., and Hiegel, J. M., J. of Research, 28, 643 (1942).

${ }^{3}$ Shushpanov, I. I., Metallurg., 12, 31 (1937).

\section{Multiple Monochromators}

IN a recent communication, Rochester and Martin ${ }^{1}$ have commented on my note ${ }^{2}$ describing the construction of multiple monochromators, and claim that two similar ideas have been suggested previously. One of these suggestions, which is merely an extension of the Littrow principle, had not been published; the other ${ }^{3}$, so far as I am aware, has not been put into practice, and it remains to be proved that the method is practicable. Neither of these proposed methods can be extended for the construction of other than double monochromators. The same limitation applies also to the modification of my method proposed by Rochester and Martin. Comments on this will be reserved until they have tested this modification and published the results obtained. 